\title{
Association of Smoking and Obesity on the Risk of Developing Primary Sjögren Syndrome: A Population-based Cohort Study
}

\author{
Luisa Servioli, Gabriel Maciel, Carlotta Nannini, Cynthia S. Crowson, Eric L. Matteson, \\ Divi Cornec, and Alvise Berti (i)
}

\begin{abstract}
Objective. To explore the role of smoking and obesity in primary Sjögren syndrome (pSS).
Methods. Olmsted County (Minnesota, USA) residents $(n=106)$ diagnosed with pSS from 2000 to 2015 were compared to 3 controls without pSS and matched for age and sex who were randomly selected from Olmsted County residents.

Results. Current smokers were less likely to be pSS cases (OR 0.34,95\% CI 0.14-0.85), while there was no association between former smoking and case/control status (OR 1.27, 95\% CI 0.80-2.03) compared to never smokers. Smoking status was not associated with antinuclear antibody, anti-SSA, anti-SSB, or rheumatoid factor positivity ( $\mathrm{p}>0.05$ ). OR for obesity was 0.79 (95\% CI 0.48-1.30). Conclusion. In this population-based study, current smoking was inversely associated with case/control status, while body mass index lacked any association. (First Release January 15 2019; J Rheumatol 2019;46:727-30; doi:10.3899/jrheum.180481)
\end{abstract}

Key Indexing Terms: SJÖGREN SYNDROME

OBESITY

From the Division of Rheumatology, and Division of Biomedical Statistics and Informatics, and Division of Epidemiology, and Division of Pulmonary and Critical Care Medicine, Department of Health Sciences Research, Mayo Clinic College of Medicine and Science, Rochester, Minnesota, USA; Autoimmune Diseases Department, Medical Clinic 1, Hospital Maciel, Montevideo, Uruguay; Department of Rheumatology, Hospital of Prato, Prato, Italy; Rheumatology Department, Brest Teaching Hospital, Brest, France; Immunology, Rheumatology, Allergy and Rare Diseases Department, San Raffaele Scientific Institute, Milan, Italy; Rheumatology Department, Santa Chiara Hospital, Trento, Italy.

Dr. Cornec received fellowship grants from the French Society of Rheumatology and from Brest University Hospital, France. This work used the resources of the Rochester Epidemiology Project, which is supported by the US National Institute on Aging of the National Institutes of Health (NIH) under Award Number R01AG034676, and Clinical and

Translational Science Awards Grant Number UL1 TR000135 from the National Center for Advancing Translational Sciences, a component of the $\mathrm{NIH}$. The content is solely the responsibility of the authors and does not necessarily represent the official views of the NIH.

L. Servioli, MD, Division of Rheumatology, Mayo Clinic College of Medicine and Science, and Autoimmune diseases Department, Medical Clinic 1, Hospital Maciel; G. Maciel, MD, Autoimmune Diseases Department, Medical Clinic 1, Hospital Maciel; C. Nannini, MD, Division of Rheumatology, Mayo Clinic College of Medicine and Science, and Department of Rheumatology, Hospital of Prato; C.S. Crowson, PhD, Division of Rheumatology, and Division of Biomedical Statistics and Informatics, Department of Health Sciences Research, Mayo Clinic College of Medicine and Science; E.L. Matteson, MD, MPH, Division of Rheumatology, and Division of Epidemiology, Department of Health Sciences Research, Mayo Clinic College of Medicine and Science; D. Cornec, MD, PhD, Division of Pulmonary and Critical Care Medicine, Mayo Clinic College of Medicine and Science, and Rheumatology Department, Brest Teaching Hospital; A. Berti, MD, Division of Pulmonary and Critical Care Medicine, Mayo Clinic College of Medicine and Science, and the Immunology, Rheumatology, Allergy and Rare Diseases Department, San Raffaele Scientific Institute, and the Rheumatology Department, Santa Chiara Hospital.

Address correspondence to Dr. E.L. Matteson, Divisions of Rheumatology and Epidemiology, Mayo Clinic College of Medicine, 200 1st St. SW, Rochester, Minnesota 55905,USA.E-mail: Matteson.eric@mayo.edu Accepted for publication October 9, 2018.

\section{SMOKING EPIDEMIOLOGY}

Primary Sjögren syndrome (pSS) is a multisystem autoimmune disease characterized by inflammatory infiltrate and progressive dysfunction of exocrine glands, especially the lachrymal and salivary glands $\mathrm{s}^{1,2,3,4}$. Smoking is considered a risk factor for the development of several rheumatic diseases, including rheumatoid arthritis (RA) and systemic lupus erythematosus (SLE) $)^{5,6}$, although it has been shown to be inversely associated with ulcerative colitis and sarcoidosis $^{7,8}$. There are a few studies that suggest that smoking may not be related to an increased pSS risk $9,10,11,12$.

Previous evidence showed that obesity may have a role in some autoimmune conditions and chronic systemic inflammation, including psoriasis, RA, and sarcoidosis ${ }^{8,13,14,15}$. The role of body mass index (BMI) has heretofore not been investigated as a potential risk factor for $\mathrm{pSS}$, to our knowledge.

To examine the relationship among smoking, BMI and pSS, we used data from a population-based incident cohort of patients with pSS with individually matched comparators from the same population.

\section{MATERIALS AND METHODS}

Case identification and ascertainment. This retrospective, population-based study used a previously identified cohort of patients with incident pSS among residents of Olmsted County, Minnesota, USA ${ }^{1,2}$. All patients were included who received a definite diagnosis of pSS in the opinion of the evaluating rheumatologists between January 1, 2000, and December 31, 2015. The date of first pSS diagnosis was collected. Each of the cases was matched to 3 comparators from among Olmsted County residents of the same age and sex but without pSS and indexed to the date of pSS diagnosis.

The information was extracted using the resources of the Research Epidemiology Project, a medical records linkage system that allows ready access to complete (inpatient and outpatient) records from all healthcare

Personal non-commercial use only. The Journal of Rheumatology Copyright @ 2019 . All rights reserved. 
providers from the local population ${ }^{16}$. This system ensures virtually complete clinical information on all clinically recognized cases of pSS in Olmsted County residents (both cases and controls). For controls, the medical files were manually reviewed to confirm their smoking status and to verify that they did not have pSS.

In Olmsted County, smoking history is routinely obtained in the medical history questionnaire completed by patients prior to appointments. Data on smoking status were collected for patients and controls 1 year prior to index date and at index date. The medical records of cases and controls were reviewed for body weight and height to calculate BMI, closest to the index date ( $\pm 1 \mathrm{yr})$. Disease activity was retrospectively collected using European League Against Rheumatism SS Disease Activity Index outcome measures ${ }^{17,18}$. The study was approved by the institutional review boards of the Mayo Clinic (16-002401) and the Olmsted Medical Center (010-OMC-16).

Statistical analyses. Data were normally distributed and descriptive statistics [means (SD), etc.] were used to summarize the data for cases and controls. Statistical procedures included chi-square test for binary variables and Student $\mathrm{t}$ test for continuous variables.

Conditional logistic regression models were used to calculate OR. For the smoking analysis, OR were calculated for the 3-group comparison of the risk of pSS between current, former, and never smokers, with never smokers as the reference group. A p value of $<0.05$ was considered statistically significant. Analyses were performed using SAS version 9.4 (SAS Institute).

\section{RESULTS}

Smoking and pSS. There were 106 incident cases of pSS and 318 controls. Baseline characteristics of cases and controls are described in Table 1.

The proportion of current smokers was lower and the proportion of former smokers higher in patients than in comparators, respectively, while the proportion of never smokers was the same in the 2 groups. One year prior to diagnosis, the proportions of current, former, and never smokers in cases and controls were about the same (Table 1).

The OR of pSS comparing current smokers with never smokers was 0.34 (95\% CI 0.14-0.85, p < 0.021; Table 2).

Table 1. Epidemiological features of subjects with incident primary Sjögren syndrome (pSS) diagnosed in Olmsted County, Minnesota, USA, from 2000 to 2015, and age- and sex-matched controls without pSS.

\begin{tabular}{|c|c|c|c|}
\hline Baseline Characteristics & Cases, $\mathrm{n}=106$ & Controls, $\mathrm{n}=318$ & $\mathrm{p}$ \\
\hline Age, yrs, mean (SD) & $59.7(15.8)$ & $59.7(15.7)$ & $>0.999$ \\
\hline Sex, female & $88(83)$ & $264(83)$ & $>0.999$ \\
\hline Ocular symptoms & $95 / 106(90)$ & - & \\
\hline Oral symptoms & $89 / 106(84)$ & - & \\
\hline Antinuclear antibody positivity & 77/104 (74) & - & \\
\hline SSA (anti-La) positivity & $78 / 101(77)$ & - & \\
\hline SSB (anti-Ro) positivity & $61 / 100(61)$ & - & \\
\hline Rheumatoid factor positivity & $50 / 90(56)$ & - & \\
\hline Hypergammaglobulinemia present & $42 / 85(49)$ & - & \\
\hline Abnormal ocular staining ${ }^{\dagger}$ & $10 / 15(67)$ & - & \\
\hline Schirmer's test $\leq 5 / 5 \mathrm{~min}$ & $7 / 9(78)$ & - & \\
\hline Abnormal salivary scintigraphy or parotid sialography & $7 / 9(78)$ & - & \\
\hline Unstimulated salivary flow $\leq 0.1 \mathrm{ml} / \mathrm{min}$ & $1 / 1(100)$ & - & \\
\hline Histopathology positivity $\$$ & $9 / 14(64)$ & - & \\
\hline Met AECG criteria & $19(18)$ & - & \\
\hline Met 2012 ACR criteria ${ }^{\dagger \dagger}$ & $18(17)$ & - & \\
\hline \multicolumn{4}{|l|}{ ESSDAI score } \\
\hline Low disease activity $(\leq 5)$ & $42(70)$ & - & \\
\hline Moderate disease activity (5-13) & $13(22)$ & - & \\
\hline High disease activity $(\geq 14)$ & $5(8)$ & - & \\
\hline Smoking status at index date* & & & 0.012 \\
\hline Never smoker & $49(46)$ & $140(45)$ & \\
\hline Former smoker & $51(48)$ & $117(38)$ & \\
\hline Current smoker & $6(6)$ & $51(17)$ & \\
\hline Smoking status 1 year prior to index date* & & & 0.005 \\
\hline Never smoker & $48(46)$ & $138(45)$ & \\
\hline Former smoker & $52(50)$ & $116(38)$ & \\
\hline Current smoker & $5(5)$ & $51(17)$ & \\
\hline BMI at index date, $\mathrm{kg} / \mathrm{m}^{2 * *}$, mean $(\mathrm{SD})$ & $27.9(7.2)$ & $29.2(7.4)$ & 0.062 \\
\hline Obesity $\left(\mathrm{BMI} \geq 30 \mathrm{~kg} / \mathrm{m}^{2}\right)$ at index date ${ }^{* *}$ & $33(32)$ & $113(37)$ & 0.318 \\
\hline
\end{tabular}

Values are the number/total number (\%) unless otherwise indicated. ${ }^{\dagger}$ Van Bijsterveld score $\geq 4$ or Rose Bengal test. ${ }^{\$}$ According to the report of the revising pathologist. ${ }^{\dagger \dagger}$ A minority of the patients fulfilled AECG (18\%) and ACR (17\%) classification criteria because the requisite tests were not performed, not because they were negative. * Among cases, smoking status at date of diagnosis and 1 year prior to diagnosis date was available in all (100\%) and in 105 (99\%) of subjects, respectively, and among controls in $308(97 \%)$ and 305 (96\%) of subjects, respectively. ** BMI within 1 year before to 1 year after date of diagnosis was available for 104 cases (98\%) and 304 controls (96\%). AECG: American-European Consensus Group; ACR: American College of Rheumatology; ESSDAI: European League Against Rheumatism SS Disease Activity Index; BMI: body mass index. 
Table 2. OR for smoking, BMI, and obesity, and risk of primary Sjögren syndrome.

\begin{tabular}{lccc}
\hline Variables & OR & $95 \% \mathrm{CI}$ & $\mathrm{p}$ \\
\hline Smoking status at index date & & & \\
Current smoker (vs never smoker) & 0.34 & $0.14-0.85$ & 0.021 \\
Former smoker (vs never smoker) & 1.27 & $0.80-2.03$ & 0.320 \\
BMI at index date, $\mathrm{kg} / \mathrm{m}^{2}$ & 0.97 & $0.94-1.01$ & 0.090 \\
Obesity $\left(\mathrm{BMI} \geq 30 \mathrm{~kg} / \mathrm{m}^{2}\right.$ ) at index date & 0.79 & $0.48-1.30$ & 0.350 \\
\hline
\end{tabular}

The reported OR are calculated by conditional logistic regression stratified by age- and sex-matched pair. BMI: body mass index.

The OR of pSS was not significantly different between former and never smokers (OR 1.27; 95\% CI 0.80-2.03).

Among patients, $65 \%$ of ever smokers (current and former smokers combined) were antinuclear antibody (ANA)positive compared with $83 \%$ of never smokers $(\mathrm{p}=0.06)$, $72 \%$ of ever smokers were SSA-positive compared with $83 \%$ of never smokers $(p=0.16), 62 \%$ of ever smokers were SSB-positive compared with $60 \%$ of never smokers $(\mathrm{p}=$ 0.78 ), and $56 \%$ of ever smokers were rheumatoid factor (RF)positive compared with $55 \%$ of never smokers $(\mathrm{p}=0.92)$.

Obesity, BMI, and pSS. Mean BMI and proportion of obesity in patients and comparators were not statistically different between patients and comparators (Table 1). The OR of pSS comparing obese with nonobese subjects was 0.79 (95\% CI 0.48-1.30; Table 2). The OR of pSS for BMI analyzed as a continuous variable approached statistical significance (OR $0.97 ; 95 \%$ CI $0.94-1.01$ ).

\section{DISCUSSION}

In this population-based incident cohort of Olmsted County (USA) residents, an inverse association was observed between current smoking status and pSS. The results are consistent with previous preliminary referral-based cohort studies of smoking in $\mathrm{pSS}^{9,10,11,12}$. In addition, this is the first study analyzing the relationship between BMI, obesity, and pSS in a population-based setting, showing that there was no association between BMI and pSS.

Although epidemiological evidence indicates smoking as a risk factor for the development of seropositive RA, and for the development of anti-dsDNA autoantibodies in $\mathrm{SLE}^{5,6}$, it is still unclear why current smokers have a lower risk of pSS. In our population-based study, results regarding smoking are consistent with the previous nested case-control or monocentric cohort observations. Two case-cohort studies showed that current smoking was associated with a reduced risk of subsequent diagnosis of $\mathrm{pSS}^{11,12}$; in one of them, duration of smoking was inversely correlated with $\mathrm{pSS}^{12}$. In the other, to be a former smoker was associated with an increased risk of $\mathrm{pSS}^{11}$. Indeed, pSS is a slowly evolving disease, and patients could quit smoking when they experience the first early symptoms of pSS, such as dry mouth or dry cough, years before the diagnosis is made. Hence, as for other retro- spective studies, the potential for reverse causality between current smoking and onset of symptoms may not be completely excluded.

A debatable effect of smoking on antibody production has been reported in pSS. A previous cross-sectional, case-control study showed an association between ANA positivity and smoking in patients with $\mathrm{pSS}^{10}$. Further, 2 studies demonstrated that anti-SSA and anti-SSB are negatively associated with smoking ${ }^{9,12}$. In contrast, our findings did not demonstrate an association between previous or current smoking status and ANA, anti-SSA, anti-SSB, and RF positivity, paralleling the results of the only other population-based pSS study on this topic ${ }^{11}$. Although the selection criteria for patients with pSS were different from those of our current study (fulfillment of the American-European Consensus Group criteria vs physician-diagnosed pSS, respectively), the percentage of seropositivity of each autoantibody was similar. In the setting of a referral center dedicated to the disease, a patient with suspected pSS will usually undergo more comprehensive evaluation and diagnostic testing, including invasive procedures that aid in establishing whether the patient fulfills classification criteria - tools developed specifically for research purposes. In a community setting, physicians do not generally use classification criteria to diagnose the disease; in general, they order invasive tests, which nonetheless have important clinical value (such as gland biopsy), only if the results would change their clinical decisions. The approach of this study reflects the actual number of patients who are diagnosed with pSS in clinical practice. There is no gold standard to define a complex disease such as pSS, and the analysis of physician-diagnosed cases can be of help in quantifying the real burden of the disease in our society.

To the best of our knowledge, this is the first population-based study to explore the relationship between BMI and pSS. The mechanisms by which obesity may lead to the development of systemic rheumatic diseases are unknown, but it represents a potentially modifiable risk factor in predisposed individuals $8,13,14,15$. In contrast to some other autoimmune diseases, no association was found between obesity or BMI and pSS. The reasons for this are speculative, possibly due to a lower level of systemic inflammation of patients with pSS compared to other systemic rheumatic diseases. The main strength of our study is that it is a population-based study of a well-defined region of the United States with complete case ascertainment and medical record availability. Moreover, our results reflect a real-life setting of patients with a physician-based diagnosis of pSS, making them relevant for routine patient care.

The limitations are those linked to the retrospective design of the study. Data were not always systematically obtained; in particular, it was not possible to perform a precise quantification of current and previous smoking status (i.e., pack/year) and to analyze the temporality between current smoking and

Personal non-commercial use only. The Journal of Rheumatology Copyright $\subset$ 2019. All rights reserved. 
onset of pSS symptoms in all the subjects, precluding more detailed analysis. Nonetheless, smoking status and BMI were available for almost all cases and controls.

Current smoking status was inversely associated with pSS, meaning current smokers were less likely to have pSS. The mechanism underlying this association is unknown. Conversely, BMI and obesity lack any association with pSS.

\section{REFERENCES}

1. Maciel G, Crowson CS, Matteson EL, Cornec D. Incidence and mortality of physician-diagnosed primary Sjogren syndrome: time trends over a 40-year period in a population-based US cohort. Mayo Clin Proc 2017;92:734-43.

2. Maciel G, Crowson CS, Matteson EL, Cornec D. Prevalence of primary Sjogren's syndrome in a US population-based cohort. Arthritis Care Res 2017;69:1612-6.

3. Seror R, Theander E, Bootsma H, Bowman SJ, Tzioufas A, Gottenberg JE, et al. Outcome measures for primary Sjogren's syndrome: a comprehensive review. J Autoimmun 2014;51:51-6.

4. Ramos-Casals M, Brito-Zerón P, Seror R, Bootsma H, Bowman SJ, Dörner T, et al. Characterization of systemic disease in primary Sjogren's syndrome: EULAR-SS task force recommendations for articular, cutaneous, pulmonary and renal involvements. Rheumatology 2015;54:2230-8.

5. Barbhaiya M, Tedeschi SK, Lu B, Malspeis S, Kreps D, Sparks JA, et al. Cigarette smoking and the risk of systemic lupus erythematosus, overall and by anti-double stranded DNA antibody subtype, in the nurses' health study cohorts. Ann Rheum Dis 2018;77:196-202.

6. Costenbader KH, Feskanich D, Mandl LA, Karlson EW. Smoking intensity, duration, and cessation, and the risk of rheumatoid arthritis in women. Am J Med 2006;119:503.e1-9.

7. Ananthakrishnan AN. Epidemiology and risk factors for IBD. Nat Rev Gastroenterol Hepatol 2015;12:205-17.

8. Ungprasert P, Crowson CS, Matteson EL. Smoking, obesity and risk of sarcoidosis: a population-based nested case-control study. Resp Med 2016;120:87-90.
9. Manthorpe R, Benoni C, Jacobsson L, Kirtava Z, Larsson A, Liedholm R, et al. Lower frequency of focal lip sialadenitis (focus score) in smoking patients. Can tobacco diminish the salivary gland involvement as judged by histological examination and anti-SSA/RO and anti-SSB/LA antibodies in Sjogren's syndrome? Ann Rheum Dis 2000;59:54-60.

10. Karabulut G, Kitapcioglu G, Inal V, Kalfa M, Yargucu F, Keser G, et al. Cigarette smoking in primary Sjogren's syndrome: positive association only with ANA positivity. Mod Rheumatol 2011; 21:602-7.

11. Olsson P, Turesson C, Mandl T, Jacobsson L, Theander E. Cigarette smoking and the risk of primary Sjogren's syndrome: A nested case control study. Arthritis Res Ther 2017;19:50.

12. Stone DU, Fife D, Brown M, Earley KE, Radfar L, Kaufman CE, et al. Effect of tobacco smoking on the clinical, histopathological, and serological manifestations of Sjogren's syndrome. PLoS One 2017;12:e0170249.

13. Crowson CS, Matteson EL, Davis JM 3rd, Gabriel SE. Contribution of obesity to the rise in incidence of rheumatoid arthritis. Arthritis Care Res 2013;65:71-7.

14. Armstrong AW, Harskamp CT, Armstrong EJ. The association between psoriasis and obesity: a systematic review and meta-analysis of observational studies. Nutr Diabetes 2012;2:e54.

15. Bhole VM, Choi HK, Burns LC, Vera Kellet C, Lacaille DV, Gladman DD, et al. Differences in body mass index among individuals with PsA, psoriasis, RA and the general population. Rheumatology 2012;51:552-6.

16. St Sauver JL, Grossardt BR, Yawn BP, Melton LJ 3rd, Rocca WA. Use of a medical records linkage system to enumerate a dynamic population over time: The Rochester Epidemiology Project. Am J Epidemiol 2011;173:1059-68.

17. Seror R, Bowman SJ, Brito-Zeron P, Theander E, Bootsma H, Tzioufas A, et al. EULAR Sjogren's syndrome disease activity index (ESSDAI): A user guide. RMD Open 2015;1:e000022.

18. Lendrem D, Mitchell S, McMeekin P, Gompels L, Hackett K, Bowman S, et al. Do the EULAR Sjogren's syndrome outcome measures correlate with health status in primary Sjogren's syndrome? Rheumatology 2015;54:655-9. 